\title{
Introducción: Estado, instituciones y políticas públicas con enfoque feminista en el análisis de viejas y nuevas desigualdades de género en América Latina Introduction: State, Institutions and Public Policies with a Feminist Approach in the Analysis of Old and New Gender Inequalities in Latin America
}

DÉBORA LOPREITE

Universidad de Buenos Aires, Argentina

deboralopreite@hotmail.com

ANA LAURA RODRÍGUEZ GUSTÁ ${ }^{1}$

Universidad Nacional de San Martín, Argentina

alrgusta@unsam.edu.ar

https://doi.org/10.46468/rsaap.15.2.I

\section{Introducción}

Este dossier reúne investigaciones originales referidas a diversas facetas de la institucionalidad de género en el Estado y de las políticas públicas en América Latina. Con diversas perspectivas teóricas y metodológicas, esta publicación convocó a investigadoras de diferentes procedencias y perfiles, quienes convergen en recuperar la relevancia del Estado y de las instituciones políticas para la comprensión de la regulación y transformación de las relaciones de género en las sociedades contemporáneas. Conjuntamente considerados, los trabajos plantean los siguientes interrogantes: ¿̇uáles son los actores comprometidos con las políticas de género y la institucionalidad de género en el Estado? ¿Son los gobiernos de izquierda favorables a las políticas públicas de igualdad de género y al feminismo de Estado? ¿Cuándo y cómo los actores conservadores logran ejercer poder de veto sobre las políticas públicas y la institucionalidad de género? ¿En qué medida las creencias culturales respecto al papel de mujeres y hombres se reflejan en las políticas públicas y qué consecuencias trae ello sobre la igualdad de oportunidades?

A continuación, se recapitulan algunas de las corrientes que han examinado la relación entre Estado y feminismo y luego se reflexiona acerca de los aportes de las autoras a estas discusiones más amplias sobre institucionalidad y relaciones de género.

La autoría está ordenada alfabéticamente. 


\section{Estado y feminismo}

Las investigaciones feministas siempre abordaron el análisis del Estado con cierta ambivalencia. El Estado ha sido criticado por su poder coercitivo e imposición de papeles tradicionales sobre las mujeres, por sus sesgos androcéntricos y alianzas con el capitalismo y el patriarcado. En la década de los ochenta, Catharine MacKinnon (1985) y Carole Pateman (1995) cuestionaron el corazón de la teoría clásica liberal al poner en el centro las desigualdades de género y los mecanismos de subordinación de las mujeres. Pateman explicó el carácter patriarcal del Estado democrático, criticando el concepto de contrato social ya que éste descansa sobre la concepción del individuo como propietario de su propia persona. Esta autora notó que individuo y contrato son categorías masculinas mientras que relegaron a las mujeres al ámbito privado. Mackinnon profundizó estas reflexiones al proponer que la sexualidad es al feminismo lo que el trabajo al marxismo. En este sentido, el Estado no solo se define por los intereses que sirve o por quienes detentan el poder, sino porque garantiza ciertas relaciones sociales que, además de facilitar los modos de producción capitalistas, conllevan relaciones generizadas de subordinación. Para Mackinnon, el Estado protege el poder masculino y garantiza el control de éste sobre las mujeres en todos los ámbitos.

En las últimas décadas, el Estado comenzó a ser recuperado desde perspectivas teóricas feministas que destacan su capacidad de reconocer y garantizar derechos (Hernes, 1987; McBride y Mazur, 1995; O’Connor et.al., 1999). Sin desconocer que, a través de sus intervenciones, el Estado regula relaciones que también producen desigualdades de género, parecería existir cierto consenso acerca de la potencialidad transformadora de las políticas públicas. Esta potencialidad transformadora se observaría en la capacidad para remover discriminaciones de género mediante la redistribución de recursos, el reconocimiento, y el aseguramiento de la representación de las mujeres en las decisiones sociales y políticas (Walby, 1990; O’Connor et.al., 1999).

Helga Hernes (1987) fue una de las pioneras en estudiar el papel de los Estados nación en la regulación de las relaciones sociales. Ella acuñó el concepto de Feminismo de Estado (State Feminism) como oposición al Estado patriarcal. Hernes asume que el Estado de bienestar escandinavo, con sus políticas de cuidado y cupos para las mujeres, se convierte en un Estado que incorpora las demandas de los movimientos de mujeres y feministas, al desarrollar políticas públicas que representan los "intereses de las mujeres". La influencia de Hernes se observa en dos corrientes que incorporan la 
perspectiva de género en el estudio del Estado: por un lado, desde la década de los noventa, autoras feministas como Sainsbury (1996) y O'Connor, Orloff y Shaver (1999) analizaron las diferentes formas que adoptan las relaciones de género, según la variedad de los Estados de bienestar. Dichas diferencias se explican por la combinación de relaciones públicas y privadas que sostienen las distintas formas de protección social destinadas a las mujeres, en particular, en la forma en que la organización de la protección social combina el trabajo remunerado de las mujeres, el trabajo no remunerado (o responsabilidades domésticas), y su acceso a servicios que permitan conciliar ambas tareas. Así encontramos tres modelos en las democracias avanzadas: conservador-familista, socialdemócrata y liberal. En el caso de América Latina, este enfoque comparado buscó dar cuenta de la particularidad de los tipos de familias, los mercados laborales formales e informales y los límites de los Estados nacionales para extender su cobertura y garantizar la protección social (Martínez Franzoni, 2008; Lopreite y McDonald, 2014).

Otra corriente, emparentada con la política comparada, es la del Feminismo de Estado que busca dar cuenta de la incorporación de femócratas y de objetivos feministas en las agencias burocráticas y en las políticas públicas, siempre y cuando respondan a las demandas y la representación de la movilización feminista. Según McBride y Mazur (1995), el feminismo de Estado apunta a identificar actividades feministas dentro del gobierno y la administración. Por lo tanto, como concepto, éste puede referirse a una variedad de situaciones: (i) cuando las políticas feministas están incluidas en diferentes estructuras burocráticas; (ii) cuando ciertas agencias incorporan objetivos feministas en las políticas públicas, y (iii) cuando se crea un mecanismo político dentro del Estado para promover los derechos de las mujeres y la igualdad de género.

A partir de las movilizaciones globalizadoras en la década de los noventa, el concepto de feminismo de Estado comenzó a utilizarse para analizar la creación de maquinarias de igualdad de género y mecanismos de avance de los derechos de las mujeres, enmarcados en las agendas internacionales de las Naciones Unidas (ONU), la Comunidad Europea y la Comisión Económica para América Latina y el Caribe (CEPAL). Tanto en Europa como en América Latina, varias investigadoras se han ocupado de examinar la calidad democrática y robustez de las agencias para las políticas de las mujeres. En estos análisis ha sido fundamental indagar en qué medida las estructuras estatales con mandato en igualdad de género ofrecen oportunidades de acción y decisión al movimiento de mujeres y el feminismo. A partir del neoinstitucionalismo de la ciencia política y la sociología, se prioriza el análisis de las características de las agencias para las mujeres, sus capacida- 
des para desarrollar políticas de mainstremaing de género y dialogar con los movimientos de mujeres. Esto último, en particular, está asociado con la calidad democrática del Estado, en la medida en que las agencias hacen más porosos los límites burocráticos a las decisiones de los movimientos sociales de mujeres y el feminismo en general (Rodríguez Gustá, 2008).

Por cierto, ningún proceso de transformación es automático y, por lo general, dependen de los equilibrios de fuerza entre actores con intereses y posiciones divergentes en materia de género y sexualidad. Como lo muestran los artículos del dossier, el Estado en tanto terreno de disputa se hace evidente en una amplia gama temática, como el aborto, la libertad sexual, la igualdad en las relaciones familiares y el cuidado de los hijos, entre otros. Estas cuestiones de género alcanzan un grado variable de visibilidad y desafían de manera diferente los legados históricos, tradiciones culturales y valores morales de estas sociedades (Htun y Weldon, 2018). Las coaliciones promotoras de las reformas feministas confrontan con actores conservadores y neoconservadores, se enfrentan a inercias estructurales que impiden consolidar estructuras institucionales y, particularmente, no pueden desconocer los cálculos instrumentales y pragmáticos de los partidos políticos que utilizan los temas de género como aquellos intercambiables.

Todas estas dinámicas son capturadas por los trabajos empíricos de este dossier. Estas dinámicas invitan a reflexionar sobre la igualdad de género como la frontera pendiente del Estado moderno, pretendidamente racional, y garante de la mentada libertad, igualdad, fraternidad o sororidad. El Estado establece derechos, define quiénes son los sujetos merecedores de asistencia social, distribuye recursos materiales, y produce valoraciones culturales con criterios de género. El dossier es oportuno dado el actual contexto de la región, donde el reagrupamiento y avance de actores conservadores -desde Brasil hasta Honduras- amenazan con revertir derechos conquistados. De hecho, la región ha sufrido reveses y retrocesos de derechos conquistados, particularmente en derechos sexuales con el advenimiento de actores neoconservadores fuertemente organizados (Zaremberg, Tabbush y Friedman, 2021).

El Estado en tanto entidad compleja y burocrática está sometido a una inercia significativa en sus maneras de "ver y hacer" (Rees, 2005), por lo cual la plena realización de la igualdad de género es lenta, costosa, y nunca lineal. Frente a esto, los procesos de construcción de institucionalidad de género bien podrían capturarse con el mito de Sísifo, ya que las reformas feministas que suponen libertades, reconocimientos de múltiples identidades y de configuraciones familiares, tienen traspiés en su aprobación e implementación. 
La institucionalidad de género ha sido estudiada desde diversas perspectivas, algunas de las cuales se recogen en este dossier. Por una parte desde las perspectivas del feminismo de Estado se ha analizado el rol de las agencias de políticas para las mujeres. Por otra parte, el papel de los sistemas políticos nacionales es clave para entender el rol de las legislaturas, los liderazgos ejecutivos y las estructuras multinivel como el federalismo en facilitar o bloquear el cambio de la legislación vigente y, en la creación de estructuras estatales para garantizar los derechos de las mujeres y las diversidades. Además, los organismos y foros internacionales como arenas de movilización feminista son relevantes en tanto promueven, aunque condicionan, los marcos de referencia de las cuestiones de género a través de su impacto en la política doméstica.

En los últimos tiempos se ha dado una expresiva expansión del feminismo que, además de mantener y diversificar su presencia social, se observa en diferentes instituciones, tanto en las propias burocracias estatales, como en las universidades, los partidos políticos y los sindicatos. Al constatar que el feminismo está "en todas partes" (Masson, 2007), la institucionalidad de género es más que un espejo de dinámicas sociales para volverse parte de una compleja trama de relaciones institucionales y sociales feministas. A pesar de esta malla de relaciones, el desarrollo de reformas feministas es un resultado incierto que requiere un esquema multicausal que incorpora las instituciones formales e informales, la saliencia del tópico en la agenda pública, la presión internacional en favor de los derechos humanos de las mujeres y los consensos domésticos. Cuando se trata de temas contraculturales altamente conflictivos, como el aborto, el feminismo enfrenta estructuras institucionales formales y poderes fácticos poderosos.

En varios regímenes presidencialistas como los latinoamericanos, el partido político en el Poder Ejecutivo concentra los recursos materiales y simbólicos para promover o inhibir transformaciones de género. Por ello es un Estado con el poder menos disperso, al menos en lo referido a su institucionalidad formal. Sin embargo, cuando se busca vincular la ideología de gobierno y las reformas feministas, los temas contraculturales son los más proclives a ser la "moneda de cambio" en la negociación instrumental. Es decir, en regímenes presidencialistas sometidos a presiones de gobernabilidad, las características de la coyuntura política, la estabilidad de las coaliciones electorales y gobernantes y la fragmentación o no de los sistemas partidarios producen configuraciones causales complejas. 


\section{Hoja de ruta}

En la primera parte del dossier, los artículos de Conny Roggeband y Débora Lopreite y Ana Laura Rodríguez Gustá, indagan en la institucionalidad de género en el Estado. En "The Return of the State and the Pink Tide in Latin America: Implications for the Capacity and Democratic Quality of Gender Equality Agendas” Conny Roggeband aborda, específicamente, el papel de gobiernos de la "marea rosa" en Argentina, Bolivia, Chile y Ecuador. Su estudio comparativo muestra que no hay una asociación directa ni lineal entre el "progresismo" de la década anterior y el fortalecimiento del feminismo de Estado. Por el contrario, se evidencian sorpresas e incluso paradojas. Por ejemplo, bajo el gobierno de Rafael Correa en Ecuador, la institucionalidad de género se debilitó tanto en sus cualidades burocráticas como en la participación de la sociedad civil de mujeres. Como contrapunto, en Bolivia, bajo el Movimiento al Socialismo (MAS), se registró un singular avance en la promoción a la participación de las mujeres organizadas, aunque este logro no implicó el fortalecimiento de una agencia especializada en igualdad de género. A su vez, el Servicio Nacional de la Mujer (SERNMAN) de Chile (antes de la creación del Ministerio de la Mujer y Equidad de Género) desplegó cualidades institucionales robustas, aunque sin mayores espacios de interlocución Estado / sociedad, produciéndose un distanciamiento con los movimientos de mujeres. Argentina, a pesar de su flamante Ministerio de Mujeres, Géneros y Diversidad, careció de un Consejo Nacional de las Mujeres con un umbral mínimo en su influencia política, capacidades y enraizamiento social. De esta forma, Roggeband concluye que los gobiernos de centro izquierda no se involucraron con la arquitectura necesaria para el feminismo de Estado, a pesar de las expectativas de los movimientos de mujeres en la región. Algunas diferencias, no obstante, aparecen cuando se toma en cuenta el tipo de partido de izquierda. Sin embargo, en términos generales, el panorama resultante confirma, nuevamente, las ambigüedades entre la agenda gubernamental de izquierda y las reformas feministas, en concordancia con otras investigaciones precedentes (Friedman y Tabbush, 2019).

El artículo "Feminismo de Estado en la Argentina democrática (19832021): modelo aspiracional o realidad institucional”, de Débora Lopreite y Ana Laura Rodríguez Gustá, presenta un estudio con perspectiva longitudinal de la agencia para las políticas para las mujeres en el país, desde el retorno a la democracia con Raúl Alfonsín en 1983 hasta nuestros días con el Ministerio de la Mujer, Géneros y Diversidad bajo Alberto Fernández. Con un enfoque relacional del Estado, las autoras encuentran que las redes 


\section{Débora Lopreite y Ana Laura Rodríguez Gustá}

de mujeres políticas y, más recientemente, un movimiento social feminista de gran escala, inciden en la institucionalidad de género en el Estado. La robustez de las agencias es importante ya que es la manifestación de la capacidad estatal para abordar la desigualdad de género. Por ejemplo, el Consejo Coordinador de Políticas para la Mujer fue un nodo movilizador para la ley de cupo que, a su vez, legitimó al novel Consejo Nacional de la Mujer. En estas interacciones, los marcos globales de género expresados tanto en las Conferencias Mundiales de las Mujeres, como en la ratificación de la Convención para la Eliminación de todas las formas de Discriminación Contra la Mujer (CEDAW, por su sigla en inglés), ofrecen una ventana de oportunidad para el avance de coaliciones con agendas de derechos, aunque los efectos sobre la institucionalidad de género disten de ser directos e inmediatos.

Las autoras muestran que la trayectoria institucional denota una construcción no lineal de sucesivas acumulaciones de capacidades, perspectivas de derechos humanos y democratización de las estructuras consultivas. En este sentido, la institucionalidad nacional revela marchas y contramarchas. Esto se evidencia en experiencias tempranas de feminismo de Estado seguidas de la cristalización de perspectivas familistas opuestas a los derechos sexuales y reproductivos de las mujeres incluso, paradójicamente, bajo administraciones progresistas. Para comprender los picos y valles de esta evolución institucional, las autoras consideran las decisiones estratégicas de los poderes ejecutivos al intentar promover, ignorar o incluso bloquear las demandas feministas.

La segunda parte del dossier coloca la lupa en el contenido de las reformas feministas y los factores institucionales que influyen en su aprobación o, por el contrario, en su rechazo e incluso contra reforma. Alba Ruibal en "Poderes Ejecutivos y derecho al aborto bajo gobiernos progresistas en América Latina: Ciudad de México y Brasil” introduce la problemática de la interrupción voluntaria del embarazo en directa vinculación con la ideología del partido de gobierno. Como advierte la autora, si bien los gobiernos afines a la izquierda han producido cambios en materia de igualdad de género, no se trataría de un efecto homogéneo para la totalidad de los derechos de las mujeres. Los derechos sexuales y reproductivos y la despenalización y legalización del aborto son asuntos que concitan disputa y confrontación. Para determinar cuándo estos gobiernos son proclives a aprobar reformas que habiliten el aborto, Ruibal invita a examinar la interacción de la ideología con otros factores políticos y sociales. Con el abordaje de dos procesos contrastantes en términos de resultados legislativos, Brasil y la Ciudad de México, la autora examinó escenarios pre y post 
electorales y la inclusión del Poder Ejecutivo para promover reformas y concluye que la coyuntura electoral es condición necesaria, pero no suficiente, para explicar por qué en la Ciudad de México se aprobó el aborto y se garantizó el acceso a los servicios en 2007, mientras que en Brasil no fue posible debatir el tema en el Congreso. Ruibal determina que es necesario considerar la relación histórica entre el Estado y la Iglesia, y los arreglos institucionales que la sostienen. Por lo tanto, al momento de promover reformas favorables a la interrupción voluntaria del embarazo, resultan de peso las consideraciones estratégicas, es decir, los cálculos electorales y de gobernabilidad de los poderes ejecutivos, así como también las tradiciones nacionales de laicidad y sus efectos en las instituciones.

La autora articula dos grandes hipótesis sustentadas en el concepto de “oportunidades políticas" respecto de la vinculación entre el Estado y las demandas de los movimientos sociales, extendiéndolas para analizar gobiernos de izquierda y su postura respecto de temas doctrinales o de alta confrontación pública. Por un lado, en contextos políticos post electorales con confrontaciones significativas entre fuerzas de derecha e izquierda, los gobiernos de izquierda estarían más inclinados a atender las demandas más radicales de los movimientos sociales, para diferenciarse de sus oponentes y fortalecer sus bases de apoyo. La dinámica de la Ciudad de México ilustra esta hipótesis. Por otro lado, en contextos preelectorales signados por la amenaza de cambio bruscos en los alineamientos partidarios, los actores políticos están inclinados a buscar apoyos de actores con influencia política, pero extrapartidarios, como las instituciones religiosas. Esta hipótesis sustenta los procesos observados en Brasil bajo Dilma Rousseff y dado un sistema partidario fragmentado.

Caroline Beer en "Políticas públicas de género y federalismo en México" aborda las dinámicas del federalismo en México en el avance de las reformas feministas, en un contexto donde los gobiernos subnacionales fueron ganando prerrogativas legales. La autora examina cuándo y cómo las entidades federativas impulsaron algunas leyes en derechos de mujeres, distinguiendo procesos de convergencia -o de adopción de iniciativas análogas entre los estados- de otros de divergencia. Las dinámicas subnacionales son fundamentales en una región que, además de tres países federales de gran escala (Argentina, Brasil y México), mostró innovaciones en materia de descentralización en países unitarios.

La convergencia en materia de violencia contra las mujeres se observa en la creación de agencias de políticas para las mujeres y en la introducción de cuotas electorales de género. No obstante, el matrimonio igualitario y la legalización del aborto muestran heterogeneidad ya que las entidades 
federativas tienen comportamientos disímiles e incluso opuestos en estos asuntos. Por cierto, aborto es el tema conflictivo por excelencia, donde los contrastes se agudizan y se evidencia una suerte de "pluralismo legal" por la dispersión de posiciones entre las diferentes entidades subnacionales. México, entonces, reúne desde legislación avanzada en la Ciudad de México y los estados de Oaxaca e Hidalgo, y posiciones conservadoras que defienden la vida desde la concepción, como en las constituciones de Baja California, San Luis de Potosí y Veracruz, entre otras. Utilizando categorías como federalización, innovación y replicación, Beer muestra que el papel de la Suprema Corte de Justicia en homogeneizar posiciones que favorecieron reformas feministas ocurre en el caso de las leyes de cuota y en matrimonio igualitario. Como bien señala la autora, el desempeño de las estructuras institucionales formales es insuficiente para comprender las trayectorias de reforma. De hecho, el papel de armonización del Poder Ejecutivo Federal y las sentencias de la Suprema Corte de Justicia solamente ocurren ante la ausencia de una oposición activa en la temática, siempre que exista cierto consenso social. La legalización del aborto por parte de la Suprema Corte de Justicia en septiembre de 2021 plantea un escenario de homogeneización legal, pero abre el interrogante sobre la heterogeneidad subnacional en su implementación.

La tercera parte del dossier aborda las políticas públicas sociales. En "Maternidad y políticas públicas en Chile en el contexto del COVID-19", Gabriela Álvarez Minte, Alejandra Ramm y Jazmine Gideon muestran la relevancia de las ideas y las concepciones culturales en la hechura de las políticas públicas, de tal forma que los sesgos androcéntricos aparecen cristalizados en las estructuras estatales y en los procedimientos de gestión. Como recuerdan las autoras, el Estado en América Latina nació imponiendo una jerarquía sexual en los hogares y las comunidades. Las autoras examinan tres políticas de Chile valiosas para la distribución de recursos y acceso a derechos en las áreas de la salud, la vivienda y el trabajo. Las tres políticas están sustentadas en una construcción cultural de las mujeres en su papel exclusivo de madres, instando la adopción de ideas maternalistas por parte de las instituciones que determinan criterios de elegibilidad y otorgamiento de beneficios. La responsabilidad por el cuidado y el ejercicio de la maternidad se interpretan de tal modo que reproducen las discriminaciones contra las mujeres porque suponen que "la protección" y el "cuidado afectuoso" de las madres es abnegado, no utilitario y sin esfuerzos o natural. Solo recientemente, en vivienda, las madres "no casadas" (solteras o convivientes) accedieron a los beneficios. Esto fue posible al ser visualizadas como vulnerables y, por ende, con escasas capacidades para el cuidado y la 
crianza de sus hijos. En materia de empleo, las políticas de incentivo tuvieron el efecto paradójico de reforzar la maternidad y promover trabajos precarios, sin modificar las instituciones del mercado laboral. Las contradicciones de estas ideas son aparentes en la regulación estatal de la salud privada, donde los seguros privados excluyen a las mujeres de la titularidad o bien elevan sus costos, siempre en vinculación con el embarazo y la maternidad, siendo un mecanismo de discriminación directa para el acceso de las mujeres a estos servicios.

Como producto del aislamiento del COVID-19, se extendieron algunas medidas siempre en función de los cuidados y la calidad de madres de las mujeres, renovando las visiones maternalistas que afectan particularmente a las mujeres con menor nivel educativo e inserción laboral informal. En suma, la investigación muestra que en condiciones de conservadurismo social y debilidades de las políticas universales en la distribución de bienes y servicios, el maternalismo deviene en una política pública marginal para las mujeres más vulneradas y solamente con un criterio de compensación, con escaso lugar para la construcción de un proyecto de vida autónomo.

Finalmente, Rianne Mahon introduce la compleja trama de la configuración global de las políticas de género con foco en el caso de la política sexual y los derechos reproductivos en una nota de investigación que cierra este número. En "Las normas globales sobre igualdad de género en disputa: el caso de la salud sexual y los derechos reproductivos", Mahon explica cómo desde los años ochenta, las feministas de distintas partes del globo se movilizaron por la definición de la salud sexual y los derechos reproductivos como un elemento central de los marcos globales. Sin embargo, un análisis más profundo pone de relieve, no solo el rol de los movimientos feministas por ampliar el concepto incluso cuestionando su caracterización heteronormativa sino también, el papel de algunos Estados nacionales conservadores y el Vaticano en limitar y/o bloquear una definición de la problemática orientada a la defensa de los derechos humanos de las mujeres y personas gestantes. Este trabajo nos recuerda la importancia de la definición de las normas globales y de los actores transnacionales que operan en diferentes escalas (internacional, regional y nacional), e inciden en la formulación de políticas públicas domésticas. 


\section{Débora Lopreite y Ana Laura Rodríguez Gustá}

\section{Referencias bibliográficas}

Friedman, E. J., y Tabbush, C. (2019). “Introduction. Contesting the Pink Tide”. En E. J. Friedman (Ed.), Seeking Rights from the Left. Gender, Sexuality, and the Latin American Pink Tide (Pp. 1-47). Durham: Duke University Press.

Hernes, H. (1987). Welfare State and Woman Power: Essays in State Feminism. Oslo: Norwegian University Press.

Htun, M., y S. L. Weldon. (2018). The Logics of Gender Justice: State Action on Women's Rights around the World. Cambridge: Cambridge University Press.

Lopreite, D. y Macdonald, L. (2014) Gender and Welfare Regimes in Latin America: Early Childhood Education and Care Policies in Argentina and Mexico. Social Politics: International Studies in Gender, State EF Society, 21(1): 80-102.

Luna, J. P., y Toro Maureira, S. (2014). Capacidad del Estado y gobernabilidad democrática en América Latina: Un acercamiento de medición y evaluación basado en el Barómetro de las Américas (Perspectivas desde el Barómetro de las Américas: 2014). Vanderbilt University. Disponible en https:/www.vanderbilt.edu/lapop/insights/IO902es.pdf. MacKinnon, C. (1985). Hacia un Teoría Feminista del Estado, Madrid: Ediciones Cátedra. Martínez-Franzoni, J. (2008). Welfare Regimes in Latin America: Capturing Constellations of Markets, Families, and Policies. Latin American Politics and Society 50-2: $67-100$.

Masson, L. (2007). Feministas en todas partes. Una etnografia de espacios y narrativas feministas en Argentina. Buenos Aires: Prometeo Libros.

McBride Stetson, D. y Mazur, A. (1995). Comparative State Feminism. Thousand Oaks: Sage Publications Ltd.

O’Connor, J., Orloff, A.S y Shaver S. (1999). States, Markets, Families: Gender, Liberalism and Social Policy in Australia, Canada, Great Britain and the United States. Cambridge: Cambridge University Press.

Pateman, C. (1995). El Contrato Sexual. Barcelona: Editorial Anthropos.

Rees, T. (2005). Reflections on the uneven development of gender mainstreaming in Europe. International Feminist Journal of Politics, 7(4): 555-574.

Rodríguez Gustá, A. L. (2008). Las políticas sensibles al género. Variedades conceptuales y desafíos de intervención. Temas y Debates: Revista Universitaria de Ciencias Sociales, 12(16): 109-130.

Sainsbury, D. (1996). Gender, Equality and Welfare States. Cambridge: Cambridge University Press.

Walby, S. (1990). Theorizing Patriarchy. Cornwall. T. J. Press Ltd, Padstow.

Zaremberg, G., Tabbush, C. y Friedman, E. J. (2021). Feminism(s) and anti-gender backlash: Lessons from Latin America. International Feminist Journal of Politics, 23(4): 527-534. 